\title{
Characterizing graphs of maximum principal ratio
}

\author{
Michael Tait and Josh Tobin*
}

October 22, 2019

\begin{abstract}
The principal ratio of a connected graph, denoted $\gamma(G)$, is the ratio of the maximum and minimum entries of its first eigenvector. Cioabă and Gregory conjectured that the graph on $n$ vertices maximizing $\gamma(G)$ is a kite graph: a complete graph with a pendant path. In this paper we prove their conjecture.
\end{abstract}

\section{Introduction}

Several measures of graph irregularity have been proposed to evaluate how far a graph is from being regular. In this paper we determine the extremal graphs with respect to one such irregularity measure, answering a conjecture of Cioabă and Gregory [5].

All graphs in this paper will be simple and undirected, and all eigenvalues are of the adjacency matrix of the graph. For a connected graph $G$, the eigenvector corresponding to its largest eigenvalue, the principal eigenvector, can be taken to have all positive entries. If $\mathbf{x}$ is this eigenvector, let $x_{\min }$ and $x_{\max }$ be the smallest and largest eigenvector entries respectively. Then define the principal ratio, $\gamma(G)$ to be

$$
\gamma(G)=\frac{x_{\max }}{x_{\min }}
$$

Note that $\gamma(G) \geq 1$ with equality exactly when $G$ is regular, and it therefore can be considered as a measure of graph irregularity.

Let $P_{r} \cdot K_{s}$ be the graph attained by identifying an end vertex of a path on $r$ vertices to any vertex of a complete graph on $s$ vertices. This has been called a kite graph or a lollipop graph. Cioabă and Gregory [5] conjectured that the connected graph on $n$ vertices maximizing $\gamma$ is a kite graph. Our main theorem proves this conjecture for $n$ large enough.

Theorem 1. For sufficiently large $n$, the connected graph $G$ on $n$ vertices with largest principal ratio is a kite graph.

We note that Brightwell and Winkler [4 showed that a kite graph maximizes the expected hitting time of a random walk. Other irregularity measures for graphs have been well-studied. Bell [3] studied the irregularity measure $\epsilon(G):=\lambda_{1}(G)-\bar{d}(G)$, the difference between the spectral radius and the average degree of $G$. He determined the extremal graph over all (not necessarily connected) graphs on $n$ vertices and $e$ edges. It is not known what the extremal connected graph is, and Aouchiche et al [2] conjectured that this extremal graph is a 'pineapple':

* Both authors were partially supported by NSF grant DMS 1362650

\{mtait, rjtobin\}@math.ucsd.edu 
a complete graph with pendant vertices added to a single vertex. Bell also studied the variance of a graph,

$$
\operatorname{var}(G)=\frac{1}{n} \sum_{v \in V(G)}\left|d_{v}-\bar{d}\right|^{2} .
$$

Albertson [1] defined a measure of irregularity by

$$
\sum_{u v \in E(G)}|d(u)-d(v)|
$$

and the extremal graphs were characterized by Hansen and Mélot $[6]$.

Nikiforov 9] proved several inequalities comparing $\operatorname{var}(G), \epsilon(G)$ and $s(G):=\sum_{v}|d(u)-\bar{d}|$. Bell showed that $\epsilon(G)$ and $\operatorname{var}(G)$ are incomparable in general [3]. Finally, bounds on $\gamma(G)$ have been given in [5, 10, 8, 7, 11].

\section{Preliminaries}

Throughout this paper $G$ will be a connected simple graph on $n$ vertices. The eigenvectors and eigenvalues of $G$ are those of the adjacency matrix $A$ of $G$. The vector $v$ will be the eigenvector corresponding to the largest eigenvalue $\lambda_{1}$, and we take $v$ to be scaled so that its largest entry is 1 . Let $x_{1}$ and $x_{k}$ be the vertices with smallest and largest eigenvector entries respectively, and if several such vertices exist then we pick any of them arbitrarily. Let $x_{1}, x_{2}, \cdots, x_{k}$ be a shortest path between $x_{1}$ and $x_{k}$. Let $\gamma(G)$ be the principal ratio of $G$. We will abuse notation so that for any vertex $x$, the symbol $x$ will refer also to $v(x)$, the value of the eigenvector entry of $x$. For example, with this notation the eigenvector equation becomes

$$
\lambda v=\sum_{w \sim v} w
$$

We will make use of the Rayleigh quotient characterization of the largest eigenvalue of a graph,

$$
\lambda_{1}(G)=\max _{0 \neq v} \frac{v^{T} A(G) v}{v^{t} v}
$$

Recall that the vertices $v_{1}, v_{2}, \cdots, v_{m}$ are a pendant path if the induced graph on these vertices is a path and furthermore if, in $G, v_{1}$ has degree 1 and the vertices $v_{2}, \cdots, v_{m-1}$ have degree 2 (note there is no requirement on the degree of $v_{m}$ ).

Lemma 2. If $\lambda_{1} \geq 2$ and $\sigma=\left(\lambda_{1}+\sqrt{\lambda_{1}^{2}-4}\right) / 2$, then for $1 \leq j \leq k$,

$$
\gamma(G) \leq \frac{\sigma^{j}-\sigma^{-j}}{\sigma-\sigma^{-1}} x_{j}^{-1} .
$$

Moreover we have equality if the vertices $x_{1}, x_{2}, \cdots, x_{j}$ are a pendant path.

Proof. We have the following system of inequalities

$$
\begin{aligned}
\lambda_{1} x_{1} & \geq x_{2} \\
\lambda_{1} x_{2} & \geq x_{1}+x_{3} \\
\lambda_{1} x_{3} \geq & x_{2}+x_{4} \\
\vdots & \vdots \\
\lambda_{1} x_{j-1} & \geq x_{j}+x_{j-2}
\end{aligned}
$$


The first inequality implies that

$$
x_{1} \geq \frac{1}{\lambda_{1}} x_{2}
$$

Plugging this into the second equation and rearranging gives

$$
x_{2} \geq \frac{\lambda_{1}}{\lambda_{1}^{2}-1} x_{3}
$$

Now assume that

$$
x_{i} \geq \frac{u_{i-1}}{u_{i}} x_{i+1} .
$$

with $u_{j}$ positive for all $j<i$. Then

$$
\lambda_{1} x_{i+1} \geq x_{i}+x_{i+2}
$$

implies that

$$
x_{i+1} \geq \frac{u_{i}}{\lambda_{1} u_{i}-u_{i-1}} x_{i+2} .
$$

where $\lambda_{1} u_{i}-u_{i-1}$ must be positive because $x_{j}$ is positive for all $j$. Therefore the coefficients $u_{i}$ satisfy the recurrence

$$
u_{i+1}=\lambda_{1} u_{i}-u_{i-1}
$$

Solving this and using the initial conditions $u_{0}=1, u_{1}=\lambda$ we get

$$
u_{i}=\frac{\sigma^{i+1}-\sigma^{-i-1}}{\sigma-\sigma^{-1}}
$$

In particular, $u_{i}$ is always positive, a fact implicitly used above. Finally this gives,

$$
x_{1} \geq \frac{u_{0}}{u_{1}} x_{2} \geq \frac{u_{0}}{u_{1}} \cdot \frac{u_{1}}{u_{2}} x_{3} \geq \cdots \geq \frac{x_{j}}{u_{j-1}}
$$

Hence

$$
\gamma(G)=\frac{x_{k}}{x_{1}}=\frac{1}{x_{1}} \leq \frac{\sigma^{j}-\sigma^{-j}}{\sigma-\sigma^{-1}} x_{j}^{-1}
$$

If these vertices are a pendant path, then we have equality throughout. [5].

We will also use the following lemma which comes from the paper of Cioabă and Gregory

Lemma 3. For $r \geq 2$ and $s \geq 3$,

$$
s-1+\frac{1}{s(s-1)}<\lambda_{1}\left(P_{r} \cdot K_{s}\right)<s-1+\frac{1}{(s-1)^{2}} .
$$

In the remainder of the paper we prove Theorem 1. We now give a sketch of the proof that is contained in Section 3 ,

1. We show that the vertices $x_{1}, x_{2}, \cdots, x_{k-2}$ are a pendant path and that $x_{k}$ is connected to all of the vertices in $G$ that are not on this path (lemma 5).

2. Next we prove that the length of the path is approximately $n-n / \log (n)$ (lemma 6 ).

3. We show that $x_{k-2}$ has degree exactly 2 (lemma 9), which extends our pendant path to $x_{1}, x_{2}, \cdots, x_{k-1}$. To do this, we find conditions under which adding or deleting edges increases the principal ratio (lemma 7 ).

4. Next we show that $x_{k-1}$ also has degree exactly 2 (lemma 11). At this point we can deduce that our extremal graph is either a kite graph or a graph obtained from a kite graph by removing some edges from the clique. We show that adding in any missing edges will increase the principal ratio, and hence the extremal graph is exactly a kite graph. 


\section{Proof of Theorem 1}

Let $G$ be the graph with maximal principal ratio among all connected graphs on $n$ vertices, and let $k$ be the number of vertices in a shortest path between the vertices with smallest and largest eigenvalue entries. As above, let $x_{1}, \cdots, x_{k}$ be the vertices of the shortest path, where $\gamma(G)=x_{k} / x_{1}$. Let $C$ be the set of vertices not on this shortest path, so $|C|=n-k$. Note that there is no graph with $n-k=1$, as the endpoints of a path have the same principal eigenvector entry. Also $\lambda_{1}(G) \geq 2$, otherwise $P_{n-2} \cdot K_{3}$ would have larger principal ratio. Finally note that $k$ is strictly larger than 1 , otherwise $x_{k}=x_{1}$ and $G$ would be regular.

Lemma 4. $\lambda_{1}(G)>n-k$.

Proof. Let $H$ be the graph $P_{k} \cdot K_{n-k+1}$. It is straightforward to see that in $H$, the smallest entry of the principal eigenvector is the vertex of degree 1 and the largest is the vertex of degree $n-k+1$. Also note that in $H$, the vertices on the path $P_{k}$ form a pendant path. By maximality we know that $\gamma(G) \geq \gamma(H)$. Combining this with lemma 2, we get

$$
\frac{\sigma^{k}-\sigma^{-k}}{\sigma-\sigma^{-1}} \geq \gamma(G) \geq \gamma(H)=\frac{\sigma_{H}^{k}-\sigma_{H}^{-k}}{\sigma_{H}-\sigma_{H}^{-1}}
$$

where $\sigma_{H}=\left(\lambda_{1}(H)+\sqrt{\lambda_{1}(H)^{2}-4}\right) / 2$.

Now the function

$$
f(x)=\frac{x^{k}-x^{-k}}{x-x^{-1}}
$$

is increasing when $x \geq 1$. Hence we have $\sigma \geq \sigma_{H}$, and so $\lambda_{1}(G) \geq \lambda_{1}(H)>n-k$.

Lemma 5. $x_{1}, x_{2}, \cdots, x_{k-2}$ are a pendant path in $G$, and $x_{k}$ is connected to every vertex in $G$ that is not on this path.

Proof. By our choice of scaling, $x_{k}=1$. From lemma 4

$$
n-k<\lambda_{1}(G)=\sum_{y \sim x_{k}} y \leq\left|N\left(x_{k}\right)\right| .
$$

Now $\left|N\left(x_{k}\right)\right|$ is an integer, so we have $\left|N\left(x_{k}\right)\right| \geq n-k+1$. Moreover because $x_{1}, x_{2}, \cdots, x_{k}$ is an induced path, we must have that $\left|N\left(x_{k}\right)\right|=n-k+1$ exactly, and hence the $N\left(x_{k}\right)=C \cup\left\{x_{k-1}\right\}$. It follows that $x_{1}, x_{2}, \cdots, x_{k-3}$ have no neighbors off the path, as otherwise there would be a shorter path between $x_{1}$ and $x_{k}$.

Lemma 6. For the extremal graph $G$, we have $n-k=(1+o(1)) \frac{n}{\log n}$.

Proof. Let $H$ be the graph $P_{j} \cdot K_{n-j+1}$ where $j=\left\lfloor n-\frac{n}{\log n}\right\rfloor$, and let $G$ be the connected graph on $n$ vertices with maximum principal ratio. Let $x_{1}, \cdots, x_{k}$ be a shortest path from $x_{1}$ to $x_{k}$ where $\gamma(G)=\frac{x_{k}}{x_{1}}$. By lemma 5 , we have

$$
\lambda_{1}(G) \leq \Delta(G) \leq n-k+1 .
$$

By the eigenvector equation, this gives that

$$
\gamma(G) \leq(n-k+1)^{k}
$$

Now, lemma 2 gives that

$$
\gamma(H)=\frac{\sigma_{H}^{j}-\sigma_{H}^{-j}}{\sigma_{H}-\sigma_{H}^{-1}},
$$


where

$$
\sigma(H)=\frac{\lambda_{1}(H)+\sqrt{\lambda_{1}(H)^{2}-4}}{2} .
$$

Now, $s-1+\frac{1}{s(s-1)}<\lambda_{1}\left(P_{r} \cdot K_{s}\right)<s-1+\frac{1}{(s-1)^{2}}$, so we may choose $n$ large enough that $\frac{n}{\log n}+1>\sigma_{H}-\sigma_{H}^{-1}>\frac{n}{\log n}$. By maximality of $\gamma(G)$, we have

$$
(n-k+1)^{k} \geq \gamma(G) \geq \gamma(H) \geq\left(\frac{n}{\log n}\right)^{n-\frac{n}{\log n}-2} .
$$

Thus, $n-k=(1+o(1)) \frac{n}{\log n}$.

For the remainder of this paper we will explore the structure of $G$ by showing that if certain edges are missing, adding them would increase the principal ratio, and so by maximality these edges must already be present in $G$. We have established that the vertices $x_{1}, x_{2}, \cdots, x_{k-2}$ are a pendant path, and so we have

$$
\gamma(G)=\frac{\sigma^{k-2}-\sigma^{-k+2}}{\sigma-\sigma^{-1}} \frac{1}{x_{k-2}}
$$

We will not add any edges that affect this path, and so the above equality will remain true. The change in $\gamma$ is then completely determined by the change in $\lambda_{1}$ and the change in $x_{k-2}$. The next lemma gives conditions on these two parameters under which $\gamma$ will increase or decrease.

Lemma 7. Let $x_{1}, x_{2}, \cdots, x_{m-1}$ form a pendant path in $G$, where $n-m=(1+o(1)) n / \log (n)$. Let $G_{+}$be a graph obtained from $G$ by adding some edges from $x_{m-1}$ to $V(G) \backslash\left\{x_{1}, \cdots, x_{m-1}\right\}$, where the addition of these edges does not affect which vertex has largest principal eigenvector entry. Let $\lambda_{1}^{+}$be the largest eigenvalue of $G_{+}$with leading eigenvector entry for vertex $x$ denoted $x^{+}$, also normalized to have maximum entry one. Define $\delta_{1}$ and $\delta_{2}$ such that $\lambda_{1}^{+}=\left(1+\delta_{1}\right) \lambda_{1}$ and $x_{m-1}^{+}=\left(1+\delta_{2}\right) x_{m-1}$. Then

- $\gamma\left(G_{+}\right)>\gamma(G)$ whenever $\delta_{1}>4 \delta_{2} / n$

- $\gamma\left(G_{+}\right)<\gamma(G)$ whenever $\delta_{1} \exp \left(2 \delta_{1} \lambda_{1} \log n\right)<\delta_{2} / 3 n$.

Proof. We have

$$
\sigma=\lambda_{1}-\lambda_{1}^{-1}-\lambda_{1}^{-3}-2 \lambda_{1}^{-5}-\cdots-\frac{2}{2 n-3}\left(\begin{array}{c}
2 n-2 \\
n
\end{array}\right) \lambda_{1}^{-(2 n-1)}-\cdots
$$

So

$$
\lambda_{1}^{+}-\lambda_{1}<\sigma_{+}-\sigma<\lambda_{1}^{+}-\lambda_{1}-2\left(\left(\lambda_{1}^{+}\right)^{-1}-\lambda_{1}^{-1}\right)
$$

when $\lambda_{1}$ is sufficiently large, which is guaranteed by lemma 6. Plugging in $\lambda_{1}^{+}=\left(1+\delta_{1}\right) \lambda_{1}$, we get

$$
\delta_{1} \lambda_{1}<\sigma_{+}-\sigma<\delta_{1} \lambda_{1}+2 \lambda_{1}^{-1}\left(1-\left(1+\delta_{1}\right)^{-1}\right)<\delta_{1} \lambda_{1}+\delta_{1}
$$

In particular

$$
\left(1+\delta_{1} / 2\right) \sigma<\sigma_{+}<\left(1+2 \delta_{1}\right) \sigma
$$

To prove part (i), we wish to find a lower bound in the change in the first factor of equation 3 Let

$$
f(x)=\frac{x^{m-1}-x^{-m+1}}{x-x^{-1}} .
$$


Then $2 m x^{m-3}>f^{\prime}(x)>(m-2) x^{m-3}-m x^{m-5}$, and using that $n-m \sim n / \log (n)$ and $\sigma \sim \lambda_{1}$ which goes to infinity with $n$, we get $f^{\prime}(x) \gtrsim(m-2) x^{m-3}$. By linearization and because $f(\sigma) \sim \sigma^{m-2}$, it follows that

$$
\frac{\sigma_{+}^{m-1}-\sigma_{+}^{-m+1}}{\sigma_{+}-\sigma_{+}^{-1}} \geq\left(1+\frac{\delta_{1}(m-3)}{2}\right) \frac{\sigma^{m-1}-\sigma^{-m+1}}{\sigma-\sigma^{-1}}
$$

Hence, if

$$
\frac{\delta_{1}(m-3)}{2}>\delta_{2}
$$

then $\gamma\left(G_{+}\right)>\gamma(G)$. In particular it is sufficient that $\delta_{1}>4 \delta_{2} / n$.

To prove part (ii), recall from above that $f^{\prime}(x)<2 m x^{m-3}$. Then, when $x=(1+$ $o(1))(n / \log (n))$

$$
\begin{aligned}
f^{\prime}(x+\varepsilon) & <2 m(x+\varepsilon)^{m-3} \\
& =2 m x^{m-3}\left(1+\frac{\varepsilon}{x}\right)^{m-3} \\
& \leq 2 m x^{m-3} \exp \left(\frac{m \varepsilon}{x}\right) \\
& \leq 2 n x^{m-3} \exp (2 \log (n) \varepsilon)
\end{aligned}
$$

So for $0<\varepsilon<\delta_{1} \lambda_{1}$, we have

$$
f^{\prime}(x+\varepsilon)<2 n x^{m-3} \exp \left(2 \log (n) \delta_{1} \lambda_{1}\right)
$$

Hence

$$
\left(1+3 n \exp \left(2 \delta_{1} \lambda_{1} \log n\right) \delta_{1}\right) \frac{\sigma^{m-1}-\sigma^{-m+1}}{\sigma-\sigma^{-1}}>\frac{\sigma_{+}^{m-1}-\sigma_{+}^{-m+1}}{\sigma_{+}-\sigma_{+}^{-1}}
$$

Lemma 8. For every subset of $U$ of $N\left(x_{k}\right)$, we have

$$
|U|-1<\sum_{y \in U} y \leq|U|
$$

An immediate consequence is that there is at most one vertex in the neighborhood of $x_{k}$ with eigenvector entry smaller than $1 / 2$.

Proof. The upper bound follows from $y \leq 1$, and the lower bound from the inequalities

$$
\sum_{y \in N\left(x_{k}\right) \backslash U} y \leq\left|N\left(x_{k}\right)\right|-|U|
$$

and

$$
\sum_{y \in N\left(x_{k}\right)} y=\lambda_{1}(G)>\left|N\left(x_{k}\right)\right|-1
$$

Lemma 9. The vertex $x_{k-2}$ has degree exactly 2 in $G$. 
Proof. Assume to the contrary. Let $U=N\left(x_{k-2}\right) \cap N\left(x_{k}\right)$. Then $|U| \geq 2$, so by lemma 8 we have

$$
\sum_{y \in U} y>|U|-1 \geq 1
$$

Now, by the same argument as the in the proof of lemma 2, we have that

$$
\gamma(G)=\frac{\sigma^{k-1}-\sigma^{-k+1}}{\sigma-\sigma^{-1}}\left(\sum_{y \in U} y\right)^{-1}
$$

Let $H=P_{k-1} \cdot K_{n-k+2}$. Then by maximality of $\gamma(G)$ we have

$$
\frac{\sigma^{k-1}-\sigma^{-k+1}}{\sigma-\sigma^{-1}}>\gamma(G) \geq \gamma(H)=\frac{\sigma_{H}^{k-1}-\sigma_{H}^{-k+1}}{\sigma_{H}-\sigma_{H}^{-1}}
$$

So $\sigma>\sigma_{H}$, which means $\lambda_{1}(G)>\lambda_{1}(H)>n-k+1$. This means that $\Delta(G)>n-k+1$, but we have established that $\Delta(G)=n-k+1$.

We now know that $x_{1}, x_{2}, \cdots, x_{k-1}$ is a pendant path in $G$, and so equation 3 becomes

$$
\gamma(G)=\frac{\sigma^{k-1}-\sigma^{-k+1}}{\sigma-\sigma^{-1}} \frac{1}{x_{k-1}}
$$

Lemma 10. The vertex $x_{k-1}$ has degree less than $11|C| / \sqrt{\log n}$.

Proof. Assume to the contrary, so throughout this proof we assume that the degree of $x_{k-1}$ is at least $11|C| / \sqrt{\log n}$. Let $G_{+}$the graph obtained form $G$ with an additional edge from $x_{k-1}$ to a vertex $z \in C$ with $z \geq 1 / 2$. Let $\lambda_{1}^{+}=\lambda_{1}\left(G_{+}\right)$and let $x^{+}$be the principal eigenvector entry of vertex $x$ in $G_{+}$, where this eigenvector is normalized to have $x_{k}^{+}=1$.

Change in $\lambda_{1}$ : By equation 1 , we have $\lambda_{1}^{+}-\lambda_{1} \geq 2 \frac{x_{k-1} z}{\|v\|_{2}^{2}}$. A crude upper bound on $\|v\|_{2}^{2}$ is

$$
\|v\|_{2}^{2} \leq 1+\sum_{y \sim x_{k}} y+\frac{2}{\lambda_{1}}+\frac{4}{\lambda_{1}^{2}}+\cdots<2 \lambda_{1}
$$

We also have that $z \geq 1 / 2$ so

$$
\lambda_{1}^{+} \geq\left(1+\frac{x_{k-1}}{2 \lambda_{1}^{2}}\right) \lambda_{1}
$$

Change in $x_{k-1}$ : Let $U=N\left(x_{k-1} \cap C\right)$. By the eigenvector equation we have

$$
\begin{aligned}
& x_{k-1}=\frac{1}{\lambda_{1}}\left(x_{k-2}+x_{k}+\sum_{y \in U} y\right) \\
& x_{k-1}^{+}=\frac{1}{\lambda_{1}^{+}}\left(x_{k-2}^{+}+x_{k}^{+}+z^{+}+\sum_{y \in U} y^{+}\right)
\end{aligned}
$$

Subtracting these, and using that $\lambda_{1}<\lambda_{1}^{+}$and $x_{k}=x_{k}^{+}=1$, we get

$$
x_{k-1}^{+}-x_{k-1} \leq \frac{1}{\lambda_{1}}\left(x_{k-2}^{+}-x_{k-2}+z^{+}+\sum_{y \in U} y^{+}-y\right) \text {. }
$$


By lemma 8 , we have $\sum_{y \in U} y^{+}-y \leq 1$. We also have $x_{k-2}^{+}-x_{k-2}<1$ and $z^{+} \leq 1$. Hence $x_{k-1}^{+}-x_{k-1} \leq 3 / \lambda_{1}$, or

$$
x_{k-1}^{+} \geq\left(1+\frac{3}{\lambda_{1} x_{k-1}}\right) x_{k-1}
$$

We can only apply lemma 7 if $x_{k}^{+}$is the largest eigenvector entry in $G_{+}$. So we must consider two cases.

Case 1: If in $G^{+}$the largest eigenvector entry is still attained by vertex $x_{k}$, then we can apply lemma 7 and see that $\gamma\left(G^{+}\right)>\gamma(G)$ if

$$
\frac{x_{k-1}}{2 \lambda_{1}^{2}} \geq \frac{12}{\lambda_{1} x_{k-1} n}
$$

or equivalently

$$
x_{k-1}^{2} \geq \frac{24 \lambda_{1}}{n} .
$$

We have that $\lambda_{1}=(1+o(1))(n-n / \log (n))$, so it suffices for

$$
x_{k-1} \geq \frac{5}{\sqrt{\log n}} .
$$

We know that

$$
x_{k-1}>\frac{|U|-1}{2 \lambda_{1}} .
$$

By assumption

$$
|U|+2=N\left(x_{k-1}\right) \geq 11|C| / \sqrt{\log n}
$$

Equation 5 follows from this, so $\gamma\left(G^{+}\right)>\gamma(G)$.

Case 2: Say the largest eigenvector entry of $G^{+}$is no longer attained by vertex $x_{k}$. It is easy to see that the largest eigenvector entry is not attained by a vertex with degree less than or equal to 2 , and comparing the neighborhood of any vertex in $C$ with the neighborhood of $x_{k}$ we can see that $x_{k} \geq y$ for all $y \in C$. So the largest eigenvector entry must be attained by $x_{k-1}$. Then equation 4 no longer holds, instead we have

$$
\gamma\left(G_{+}\right)=\frac{\sigma_{+}^{k-1}-\sigma_{+}^{-k+1}}{\sigma_{+}-\sigma_{+}^{-1}} .
$$

Recall that in lemma 7 we determined the change from $\gamma\left(G_{+}\right)$to $\gamma(G)$ by considering $\lambda_{1}^{+}-\lambda_{1}$ and $x_{k-1}^{+}-x_{k-1}$. In this case, by (6), we must consider $\lambda_{1}^{+}-\lambda_{1}$ and $1-x_{k-1}$. Now if $x_{k-1}^{+}>x_{k}^{+}$ , then vertex $x_{k-1}$ in $G$ is connected to all of $C$ except perhaps a single vertex. Hence in $G$, the vertex $x_{k-1}$ is connected to all of $C$ except at most two vertices. This gives the bound

$$
1-x_{k-1} \leq 3 / \lambda_{1}
$$

and so as in the previous case, $\gamma\left(G_{+}\right)>\gamma(G)$.

So in all cases, $x_{k-1}$ is connected to all vertices in $C$ that have eigenvector entry larger than $1 / 2$. If all vertices in $C$ have eigenvector entry larger than $1 / 2$, then $x_{k-1}$ is connected to all of $C$, and this implies that $x_{k-1}>x_{k}$, which is a contradiction. At most one vertex in $C$ is smaller than $1 / 2$, and so there is a single vertex $z \in C$ with $z<1 / 2$. We will quickly check that adding the edge $\left\{x_{k-1}, z\right\}$ increases the principal ratio. As before let $G_{+}$be the graph obtained by adding this edge. The largest eigenvector entry in $G_{+}$is attained by $x_{k-1}$, as its neighborhood strictly contains the neighborhood of $x_{k}$. As above, adding the edge $\left\{z, x_{k}\right\}$ increases the spectral radius at least

$$
\lambda_{1}^{+}>\left(1+\frac{z}{2 \lambda_{1}^{2}}\right) \lambda_{1}
$$


and we have $1-x_{k-1}<1-z / \lambda_{1}$. Applying lemma 7 we see that $\gamma\left(G_{+}\right)>\gamma(G)$, which is a contradiction. Finally we conclude that the degree of $x_{k-1}$ must be smaller than $11|C| / \sqrt{\log n}$.

We note that this lemma gives that $x_{k-1}<1 / 2$ which implies that any vertex in $C$ has eigenvector entry larger than $1 / 2$.

Lemma 11. The vertex $x_{k-1}$ has degree exactly 2 in $G$. It follows that $x_{k-1}<2 / \lambda_{1}$.

Proof. Let $U=N\left(x_{k-1}\right) \cap C, c=|U|$. If $c=0$ then we are done. Otherwise let $G_{-}$be the graph obtained from $G$ by deleting these $C$ edges. We will show that $\gamma\left(G_{-}\right)>\gamma(G)$.

(1) Change in $\lambda_{1}$ : We have by equation 1

$$
\lambda_{1}-\lambda_{1}^{-} \leq 2 c \frac{x_{k-1}}{\|v\|_{2}^{2}}
$$

By Cauchy-Schwarz,

$$
\|v\|_{2}^{2}>\sum_{x \in N\left(x_{k}\right)} x^{2} \geq \frac{\left(\sum_{x \in N\left(x_{k}\right)} x\right)^{2}}{|C|+1} \geq \frac{(n-k)^{2}}{n-k+1}
$$

We also have

$$
x_{k-1} \leq \frac{c+2}{\lambda_{1}}
$$

Combining these we get

$$
\lambda_{1}-\lambda_{1}^{-}<\frac{9 c^{2}}{\lambda_{1}(n-k+1)} \Rightarrow \lambda_{1}<\left(1+\frac{9 c^{2}}{\lambda_{1} \lambda_{1}^{-}(n-k+1)}\right) \lambda_{1}^{-}
$$

We have $\lambda_{1} \lambda_{1}^{-}>(n-k)^{2}$, so

$$
\lambda_{1}<\left(1+\frac{10 c^{2}}{(n-k)^{3}}\right) \lambda_{1}^{-}
$$

(2) Change in $x_{k-1}$ : At this point, we know that in $G_{-}$the vertices $x_{1}, \cdots, x_{k}$ form a pendant path, and so by the proof of lemma 2, we have $x_{k-1}^{-}=(1+o(1)) / \lambda_{1}$. By the eigenvector equation and using that the vertices in $C$ have eigenvector entry at least $1 / 2$, we have $x_{k-1}>(1+c / 2) / \lambda_{1}$. So

$$
x_{k-1}-x_{k-1}^{-}>\frac{1}{\lambda_{1}}\left(\frac{c}{2}+o(1)\right)
$$

In particular,

$$
x_{k-1}>\left(1+\frac{c}{3 x_{k-1}^{-} \lambda_{1}}\right) x_{k-1}^{-}
$$

Applying lemma 7] it suffices now to show that

$$
\frac{10 c^{2}}{(n-k)^{3}} \exp \left(2 \frac{10 c^{2}}{(n-k)^{3}} \lambda_{1}^{-} \log n\right)<\frac{c}{9 x_{k-1}^{-} \lambda_{1} n} .
$$

Now

$$
\frac{10 c^{2}}{(n-k)^{3}}<10 \frac{11^{2}}{\log (n)} \frac{|C|^{2}}{(n-k)^{3}}<\frac{11^{3}}{\log n} \frac{\log n}{n}=\frac{11^{3}}{n} .
$$


Similarly $2 \frac{10 c^{2}}{(n-k)^{3}} \lambda_{1}^{-} \log n<2 \cdot 11^{3}$, so the lefthand side of equation 7 is smaller than $C_{0} / n$, where $C_{0}$ is an absolute constant. For the righthand side, recall that $x_{k-1}^{-} \lambda_{1}=1+o(1)$, and also that

$$
c>\frac{11}{\sqrt{\log n}}\left(\frac{n}{\log n}+o(1)\right)>\frac{10 n}{\log ^{3 / 2} n} .
$$

So the righthand side is larger than $1 / \log ^{3 / 2} n$. Hence for large enough $n$, the righthand side is larger than the lefthand side.

We are now ready to prove the main theorem.

Theorem 1. For sufficiently large $n$, the connected graph $G$ on $n$ vertices with largest principal ratio is a kite graph.

Proof. It remains to show that $C$ induces a clique. Assume it does not, and let $H$ be the graph $P_{k} \cdot K_{n-k+1}$. We will show that $\gamma(H)>\gamma(G)$, and this contradiction tells us that $C$ is a clique. As before, lemma 2 gives that

$$
\gamma(H)=\frac{\sigma_{H}^{k}-\sigma_{H}^{-k}}{\sigma_{H}-\sigma_{H}^{-1}}
$$

where

$$
\sigma(H)=\frac{\lambda_{1}(H)-\sqrt{\lambda_{1}(H)^{2}-4}}{2} .
$$

Since $x_{1}, \cdots x_{k}$ form a pendant path we also know that

$$
\gamma(G)=\frac{\sigma^{k}-\sigma^{-k}}{\sigma-\sigma^{-1}}
$$

Now, $\lambda_{1}(H)>\lambda_{1}(G)$ because $E(G) \subsetneq E(H)$. Since the functions $g(x)=x+\sqrt{x^{2}-4}$ and $f(x)=\left(x^{k}-x^{-k}\right) /\left(x-x^{-1}\right)$ are increasing when $x \geq 1$, we have $\gamma(H)>\gamma(G)$.

\section{Acknowledgements}

We would like to thank greatly Xing Peng for helpful discussions and comments on an earlier draft of this paper.

\section{References}

[1] Michael O Albertson. The irregularity of a graph. Ars Combinatoria, 46:219-225, 1997.

[2] Mustapha Aouchiche, Francis K Bell, D Cvetković, Pierre Hansen, Peter Rowlinson, SK Simić, and D Stevanović. Variable neighborhood search for extremal graphs. 16. some conjectures related to the largest eigenvalue of a graph. European Journal of Operational Research, 191(3):661-676, 2008.

[3] Francis K Bell. A note on the irregularity of graphs. Linear Algebra and its Applications, 161:45-54, 1992.

[4] Graham Brightwell and Peter Winkler. Maximum hitting time for random walks on graphs. Random Structures $\& 3$ Algorithms, 1(3):263-276, 1990.

[5] Sebastian M Cioabă and David A Gregory. Principal eigenvectors of irregular graphs. Electron. J. Linear Algebra, 16:366-379, 2007. 
[6] Pierre Hansen, Hadrien Mélot, and Québec) Groupe d'études et de recherche en analyse des décisions (Montréal. Variable neighborhood search for extremal graphs 9: Bounding the irregularity of a graph. Montréal: Groupe d'études et de recherche en analyse des décisions, 2002.

[7] Geoff A Latham. A remark on minc's maximal eigenvector bound for positive matrices. SIAM Journal on Matrix Analysis and Applications, 16(1):307-311, 1995.

[8] Henryk Minc. On the maximal eigenvector of a positive matrix. SIAM Journal on Numerical Analysis, 7(3):424-427, 1970.

[9] Vladimir Nikiforov. Eigenvalues and degree deviation in graphs. Linear Algebra and its Applications, 414(1):347-360, 2006.

[10] Britta Papendieck and Peter Recht. On maximal entries in the principal eigenvector of graphs. Linear Algebra and its Applications, 310(1):129-138, 2000.

[11] Xiao-Dong Zhang. Eigenvectors and eigenvalues of non-regular graphs. Linear Algebra and its Applications, 409:79-86, 2005. 